\title{
EL DERECHO A LA AUTONOMÍA DE LOS PACIENTES EN LOS CUIDADOS INTENSIVOS NEONATALES DE LA REGIÓN DE MURCIA
}

\author{
Maa DOLORES RODRÍGUEZ RABADÁN \\ Enfermera en UCI Neonatal del Hospital Virgen de la Arrixaca \\ Especialista en Pediatría. Máster en Bioderecho. Doctora en Enfermería \\ mdolores.rodriguez2@um.es
}

RESUMEN: Las prácticas sanitarias realizadas en Unidades de Cuidados Intensivos Neonatales (UCIN) se caracterizan por realizarse frecuentemente en situaciones de urgencia vital con pacientes que no pueden decidir por sí mismos. Estas peculiaridades propician que los profesionales sanitarios no identifiquen el derecho del paciente a su autonomía en numerosas ocasiones. Al mismo tiempo, la regulación de este derecho mediante la ley básica 41/2002 y su consecuente desarrollo legislativo, proporciona una terminología variada y heterogénea. Desde la perspectiva de los cuidados, se analiza en la legislación las figuras del médico responsable y el enfermero responsable, haciendo una lectura crítica y minuciosa de la regulación tanto de la información como el consentimiento informado en las UCINs en la Región de Murcia.

PALABRAS ClAVE: autonomía, información, consentimiento informado y neonato.

ABSTRACT: Health practices carried out in Neonatal Intensive Care Units (NICU) are characterised by the fact that they are frequently carried out in vital emergency situations with patients who cannot decide for themselves. These peculiarities lead health professionals not to identify the patient's right to autonomy on numerous occasions. At the same time, the regulation of this right by means of basic law 41/2002 and its consequent legislative development, provides a varied and heterogeneous terminology. From the perspective of care, the figures of the responsible doctor and the responsible nurse are analysed in legislation, making a critical and detailed reading of the regulation of both information and informed consent in NICUs in the Region of Murcia.

KEYWORDS: autonomy, information, informed consent and neonate.

SUMARIO: I. EL RESPETO DE LA AUTONOMÍA DEL PACIENTE EN LAS PRÁCTICAS SANITARIAS - II. LA FIGURA DEL MÉDICO RESPONSABLE DEL PACIENTE EN LA LEGISLACIÓN - III. CARACTERÍSTICAS DEL ACCESO A LA INFORMACIÓN Y AL CONSENTIMIENTO INFORMADO EN LOS CUIDADOS INTENSIVOS NEONATALES EN LA REGIÓN DE MURCIA - III.1 El receptor de la información sanitaria del neonato - III.2 Los profesionales sanitarios que deben informar en una UCIN - III.3 El momento adecuado para proporcionar información a los padres - III.4 Las características de la información en una UCIN - III.5 Los contenidos de la información - IV. CONCLUSIONES - V. REFERENCIAS BIBLIOGRÁFICAS - VI. NORMATIVA.

\section{EL RESPETO DE LA AUTONOMÍA DEL PACIENTE EN LAS PRÁCTICAS SANITARIAS}

Las prácticas sanitarias requieren un profundo respeto de la autonomía del paciente, exigido tanto en la legislación como en la deontología de las profesiones sanitarias. Las herramientas que hacen posible el ejercicio de la autonomía de los pacientes en el ámbito de los cuidados son sus derechos a: recibir información y otorgar su voluntario consentimiento. 
La Ley General de Sanidad de 1986 fue la primera norma en reconocer los derechos de los pacientes, sin embargo, fue la Ley 41/2002, de 14 de noviembre, básica reguladora de la autonomía del paciente y de derechos y obligaciones en materia de información y documentación clínica, la que específicamente recoge como uno de sus objetivos, reforzar y dar un trato especial al derecho de autonomía del paciente (Sánchez-Caro y Abellán, 2003, p.45). Esta ley establece que todos los profesionales sanitarios están obligados a informar a sus pacientes de forma adecuada, veraz, comprensible y verbalmente (por regla general); considera, también, la posibilidad de que los pacientes puedan negarse a recibirla. También regula el derecho del paciente a otorgar su voluntario consentimiento una vez informado (el denominado, consentimiento informado o CI). Éste será verbal por regla general y por escrito en los supuestos que especifica la ley: intervenciones quirúrgicas, procedimientos diagnósticos y terapéuticos invasores y, en general, aplicación de procedimientos que supongan riesgos o inconvenientes de notoria y previsible repercusión negativa sobre la salud del paciente. Como afirman Simón y Júdez (2004, p. 39), aporta el sustrato jurídico más explícito y directo sobre la teoría del consentimiento informado [a nivel nacional].

La ley básica, en su Disposición Adicional Primera, insta a las Comunidades Autónomas a adoptar las medidas necesarias para que hagan efectivos sus contenidos. En respuesta a esta disposición, la Región de Murcia publica la Ley 3/2009, de 11 de mayo, en la que realiza su propia regulación del derecho de los pacientes a su autonomía respetando los contenidos de la ley básica e incorporando algunos aspectos novedosos.

En respuesta a la legislación vigente, no podemos considerar la obtención del consentimiento sin un intercambio previo de información entre los profesionales sanitarios y el paciente. Información y consentimiento conforman un binomio indivisible e imprescindible para hacer efectivo el derecho de las personas a su autonomía en el ámbito de las prácticas sanitarias. Como afirma Galán Cortés (2014, p. 548), la autonomía del paciente forma parte del contenido del derecho fundamental a la integridad personal, física y sobre todo moral, reconocido en el artículo 15 de la Constitución Española.

\section{LA FIGURA DEL MÉDICO RESPONSABLE DEL PACIENTE EN LA LEGISLACIÓN}

La ley 41/2002 establece la figura del "médico responsable" en su artículo 4.3 como garante del cumplimiento del derecho a la información; y así lo reitera la ley 3/2009 en su artículo 11.1.j). Información que facilitará la participación activa de los pacientes en la toma de decisiones terapéuticas que afecten a su persona. Independientemente de que todos los profesionales sanitarios que intervengan en el proceso asistencial también sean responsables de informar al paciente de sus actos.

A este respecto, el desarrollo legislativo autonómico trata a esta figura de forma heterogénea:

- Han mantenido la figura del médico responsable en su desarrollo legislativo: Canarias, Cantabria, Castilla y León, Cataluña, Comunidad de Madrid, Comunidad Valenciana, Extremadura, Galicia, Islas Baleares, Principado de Asturias y Región de Murcia.

Han reconocido, además, la figura del enfermero responsable: Castilla y León, Extremadura y Región de Murcia. 
- Han eliminado la figura del médico responsable de su legislación: Andalucía (profesionales sanitarios), Castilla-La Mancha (profesional sanitario) y País Vasco (profesional sanitario y/o personal sanitario).

- Han seguido otras opciones: Aragón y La Rioja han cambiado el término por el de médico o equipo de médicos responsables (introduciendo la idea de una responsabilidad compartida). La Rioja vincula el término anterior a los documentos de CI, para lo relacionado con la información del paciente utiliza equipo sanitario. La Comunidad Foral de Navarra utiliza indistintamente, como si fueran sinónimos, los términos médico responsable y profesional sanitario responsable.

En algunas comunidades autónomas, cuando los profesionales sanitarios realizan "técnicas" a los pacientes, sólo deben proporcionarles información, no vinculando las técnicas a la utilización de los documentos de CI. Así lo legisla: Aragón, Cantabria, Castilla y León, Cataluña, Extremadura, Galicia e Islas Baleares. Sólo la Región de Murcia introduce la exigencia del consentimiento, expreso y por escrito, para "técnicas" que conlleven riesgos e inconvenientes de notoria y previsible repercusión negativa sobre la salud del paciente, además de los requerimientos ya existentes: intervenciones quirúrgicas, procedimientos diagnósticos y terapéuticos invasores y procedimientos en general.

Además, la ley básica sólo introduce indicaciones para el facultativo y médico responsable cuando trata, en su artículo 10, las condiciones de la información y consentimiento por escrito. De esta manera, da a entender que el CI verbal está dirigido a todos los profesionales sanitarios y el CI escrito sólo se debe utilizar para las actividades sanitarias realizadas por los médicos. Sin embargo, el reconocimiento del enfermero responsable llevado a cabo en algunas comunidades autónomas y la introducción de los documentos de CI para la realización de "técnicas" que se ajusten a los supuestos que indica la ley básica (art. 42.2 de la ley 3/2009 de la Región de Murcia) parece reconocer el acceso de los profesionales de Enfermería a los documentos de CI.

\section{CARACTERÍSTICAS DEL ACCESO A LA INFORMACIÓN Y AL CONSENTIMIENTO INFORMADO EN LOS CUIDADOS INTENSIVOS NEONATALES EN LA REGIÓN DE MURCIA}

La regulación del derecho a la autonomía de los recién nacidos en la Región de Murcia está marcada por la Ley 41/2002, de 14 de Noviembre, básica reguladora de la autonomía del paciente, de ámbito estatal; y la Ley 3/2009, de 11 de Mayo, de los derechos y deberes de los usuarios del sistema sanitario, de ámbito autonómico. En el capítulo III de la ley 3/2009, se especifica que los recién nacidos son un "colectivo que merece especial protección" y que éstos tendrán derecho a recibir un "tratamiento respetuoso y digno desde su nacimiento" (artículo 16).

Las prácticas sanitarias en las Unidades de Cuidados Intensivos Neonatales (en adelante, UCIN) son especialmente peculiares. La forma de proporcionar información y obtener el consentimiento informado ante cualquier intervención o técnica difieren del contexto sanitario del paciente adulto.

Cuando el paciente es un niño recién nacido, la persona o personas que responde de su autonomía son aquellas que tengan la patria potestad o tutores (normalmente serán los padres). Teniendo en cuenta que el modelo familiar no siempre es el tradicionalmente reconocido, los 
profesionales sanitarios debemos saber, en cada caso, quién es la persona que debe recibir la información y actuar en nombre del neonato.

Asimismo, las actuaciones sanitarias en las UCINs no siempre se realizan en situación de urgencia. En aquellas situaciones programadas o que aceptan demora, deben quedar en un segundo plano las actitudes paternalistas. Por tanto, las técnicas invasivas que conllevan riesgos o inconvenientes de notoria y previsible repercusión negativa sobre la salud, requieren la exigencia legal del consentimiento por escrito del progenitor (o representante legal) competente y previamente informado (Rodríguez, 2017, p. 14-15).

\section{III.1 El receptor de la información sanitaria del neonato}

El titular del derecho a la información es el paciente, así lo expresa la ley 41/2002 en su art. $5.1 \mathrm{y}$ la ley $3 / 2009$ en su art. 31.1. No obstante, "toda persona tiene derecho a que se respete su voluntad de no ser informada", siempre y cuando no sufra perjuicio el propio paciente, terceros, la colectividad y por las exigencias terapéuticas del caso (art. 4.1 y art. 9.1 de la ley 41/2002).

Cuando el paciente es un recién nacido deberá informarse plenamente a los padres no privados de la patria potestad o, en su caso, a los tutores (artículo 31.3 de la Ley 3/2009 de la Región de Murcia).

La ley 41/2002 dispone que:

- Bajo criterio médico, cuando los padres carezcan de la capacidad de entender la información a causa de su estado físico o psíquico, ésta se pueda proporcionar a personas vinculadas a ellos por razones familiares o de hecho (art. 5.3). De tal manera que, durante el ingreso de un recién nacido en una UCIN, como situación con alto nivel de estrés, los padres podrán estar acompañados si así lo deciden.

- En el caso de padres con discapacidad, la información sobre su hijo deberá proporcionarse igualmente, pero adaptada a sus posibilidades de comprensión, y además se deberá informar al representante legal del discapacitado. A este respecto se produce una adaptación normativa a la Convención Internacional sobre los Derechos de las Personas con Discapacidad mediante la Ley 26/2011 aunque, en esencia, reitera los contenidos de la ley 41/2002.

- Ante un "estado de necesidad terapéutica" (art. 5.4), limita este derecho. Es decir, establece la facultad del médico para no informar cuando conozca objetivamente que la información puede perjudicar gravemente la salud del destinatario de la misma. El médico debe dejar constancia en la historia clínica del paciente de los motivos de su decisión y comunicarlos a las personas vinculadas a los padres por razones familiares o de hecho.

La ley 3/2009, enuncia todo lo anterior y especifica claramente como excepciones al derecho de información (art. 32): la voluntad expresa de no ser informado, la situación de necesidad terapéutica y las situaciones de urgencia vital.

Asimismo, las últimas reformas legislativas sobre el consentimiento por representación dirigidas a la infancia y adolescencia de las leyes: Ley 26/2015 de 28 de Julio; Ley 8/2015, de 22 de Julio; y Ley 11/2015, de 21 de Septiembre, ejercen una doble protección en el caso del 
paciente recién nacido. Protegen, por un lado, al recién nacido como menor que no puede ejercer la titularidad del derecho a la información y, por otro, al progenitor en el caso de discapacidad y/o minoría de edad.

\section{III.2 Los profesionales sanitarios que deben informar en una UCIN}

La ley 41/2002 establece que todo profesional que interviene en la actividad asistencial está obligado a cumplir con los deberes de información (art. 1.6), aunque, es el médico responsable el garante del derecho a la información (art. 4.3).

De esta manera, el médico responsable debe garantizar que todos los profesionales que atiendan al neonato durante el proceso asistencial o le apliquen una técnica o un procedimiento concreto, cumplan con su responsabilidad de informar a los padres (art. 4.3 de la ley nacional y art. 33.1 de la regional).

En este sentido, la ley 3/2009 de la Región de Murcia hace un reconocimiento específico de los profesionales de Enfermería en dos puntos concretos de su articulado:

- Art. 11.1.j) [...] Se le asignará un enfermero en los casos en que proceda.

- Art. 33.2) [...] deberán asignar a cada paciente un médico responsable, que será su interlocutor principal con el equipo asistencial y, en su caso, un enfermero responsable del seguimiento de su plan de cuidados $[\ldots]$.

Este reconocimiento de los profesionales de Enfermería también lo encontramos en el desarrollo legislativo autonómico de Castilla y León (Ley 8/2003, de 8 de Abril) y de Extremadura (Ley 3/2005, de 8 de Julio).

\section{III.3 El momento adecuado para proporcionar información a los padres}

La Ley 41/2002 establece que:

- La información es la herramienta que se debe ofrecer al paciente para que pueda decidir libremente (art. 2.3).

- La información es el requisito previo necesario para poder otorgar el consentimiento libre y voluntario (art. 8.1).

Por tanto, la información debe ser recibida por las personas que ostenten la patria potestad o sean tutores legales del neonato antes de tomar decisiones y/o antes de recabar su CI. El consentimiento será verbal por regla general (art. 8.2) y por escrito en los supuestos que especifica la ley (art. 10).

De acuerdo con lo anterior, la ley 3/2009, en su art. 43.1, describe detalladamente el contexto en el que se debe proporcionar la información previa al consentimiento:

[...] Salvo en supuestos de urgencia, con la debida antelación y, preferentemente, no en la misma sala en donde se deba practicar la actuación asistencial, a fin de que el paciente (o su representante legal) pueda reflexionar y, en su caso, solicitar cuantas aclaraciones considere necesarias para adoptar una decisión. 
En consecuencia, el hecho de dar información no es algo puntual que ha de ocurrir en un momento determinado (Rubio, 2013, p.139). Como afirma Osuna (2013, p. 99), el cumplimiento de la obligación de informar, más allá de ser un requisito para la obtención del consentimiento informado, debe entenderse como un proceso continuado, presente y mantenido durante toda la actividad asistencial.

\section{III.4 Las características de la información en una UCIN}

Los profesionales sanitarios deben informar de forma verdadera, comprensible y adecuada a las necesidades de los padres del recién nacido, de forma que ayude a tomar decisiones de acuerdo a su propia y libre voluntad (art. 4.2 de la Ley 41/2002 y art. 30.2 de la Ley $3 / 2009$ ).

Como regla general se proporcionará verbalmente dejando constancia en la historia clínica (art. 4.1 de la ley 41/2002 y art. 30.1 de la ley 3/2009).

La ley 3/2009 de la Región de Murcia desarrolla con especial interés la forma en la que los profesionales sanitarios deben proporcionar información:

- Art. 11.1.g): especifica como derecho básico del paciente "[...] ser informado de forma comprensible, suficiente y adecuada sobre su estado de salud, y sobre las distintas opciones de técnicas diagnósticas, terapéuticas y/o farmacológicas que puedan existir en relación a su proceso asistencial".

- Art. 41.1: vuelve a reivindicar el respeto del principio de libertad y autonomía de los padres quienes, tras recibir una adecuada información asistencial sobre el proceso de su hijo, podrán decidir libremente entre las opciones clínicas o terapéuticas disponibles.

- Art. 43.1: redunda que la información previa al consentimiento informado debe ser comprensible y suficiente.

Cómo hay que informar cobra especial relevancia cuando los padres tienen alguna discapacidad. Los profesionales sanitarios deben facilitar su participación en la toma de decisiones (en la medida de lo posible), ofrecerles las medidas de apoyo pertinentes e información en formatos adecuados para que les resulten accesibles y comprensibles. Así lo establece el art. 9.5 de la Ley 41/2002 y su posterior regulación: Ley 26/2011, de adaptación normativa a la Convención Internacional sobre los Derechos de las Personas con Discapacidad; y Ley Orgánica 8/2015 y Ley 26/2015, ambas sobre la modificación del sistema de protección a la infancia y a la adolescencia.

Por tanto, el proceso de comunicación deberá ser especialmente tratado por todos los profesionales sanitarios y, en ningún caso, deberá omitirse.

\section{III.5 Control por parte de los afectados.}

La información debe constar de aquellos contenidos que ayuden a decidir libremente entre las opciones clínicas disponibles (art. 2.3 de la Ley 41/2002). La información versará sobre el conjunto del proceso asistencial: diagnóstico, pronóstico y, en su caso, alternativas de tratamiento (art. 30.1 de la Ley 3/2009). 
La ley 41/2002 diferencia entre:

- "Información asistencial" (art. 4.1), cuyos contenidos son: la finalidad y la naturaleza de cada intervención, sus riesgos y sus consecuencias (así lo reitera la Ley 3/2009 en su art. 30.1).

- Y otra información denominada "información básica", la que se debe proporcionar al paciente antes de recabar su consentimiento escrito (art. 10.1):

a) Las consecuencias relevantes o de importancia que la intervención origina con seguridad.

b) Los riesgos relacionados con las circunstancias personales $\mathrm{o}$ profesionales del paciente.

c) Los riesgos probables en condiciones normales, conforme a la experiencia y al estado de la ciencia o directamente relacionados con el tipo de intervención.

d) Las contraindicaciones.

(Ley 41/2002, de 14 de Noviembre).

Esta "información básica" la ley 3/2009 de la Región de Murcia la denomina "información esencial" y amplía sus contenidos en dos ítems, quedando de la siguiente manera:

a) Denominación y descripción básica del procedimiento quirúrgico o técnico.

b) Objetivo y resultado que se pretende alcanzar.

c) Alternativas posibles a la intervención o procedimiento propuesto.

d) Riesgos previsibles y frecuentes del procedimiento o intervención en condiciones normales de realización, conforme a la experiencia y al estado de la ciencia. En su caso, también se informará de aquellos riesgos que siendo infrecuentes, pero no excepcionales, tengan la consideración clínica de muy graves.

e) Consecuencias probables que va a originar su realización y, en su caso, aquellas que se producirían en el supuesto de no practicarse.

f) Riesgos y consecuencias previsibles en función del estado clínico y personal del paciente.

g) Contraindicaciones: circunstancias que, pudiendo sobrevenir antes de realizar el procedimiento propuesto, sean motivo de su contraindicación".

(Ley 3/2009, de 11 de Mayo)

Finalmente, ambos documentos legales recogen la posibilidad de que el paciente manifieste su deseo de no ser informado (debe quedar constancia escrita), sin perjuicio de la 
obtención de su consentimiento previo para un acto sanitario (art. 9.1 de la Ley 41/2002 y art. 45.1 de la Ley 3/2009).

Por tanto, informar es un acto de comunicación personalizado que exige habilidades y dedicación por parte de los profesionales sanitarios.

\section{CONCLUSIONES}

Los profesionales sanitarios que desempeñan su labor en las UCINs deben respetar el derecho a la autonomía del neonato a través de la participación activa de sus padres (no privados de la patria potestad) o tutores en las decisiones relativas a la salud del recién nacido.

La información se dirige a unos padres que poseen unas características específicas: altos niveles de estrés (ingreso inesperado, recién nacidos graves, riesgo vital mantenido en el tiempo, etcétera), deterioro físico de las madres (mujeres intervenidas por cesárea, puerperio complicado, enfermedades perinatales previas, etcétera), agotamiento físico y psicológico (ingresos de larga duración, en ocasiones, varios meses), etcétera. Por tanto, cuando el recién nacido está ingresado en una UCIN las condiciones en las que se debe proporcionar la información son especialmente sensibles, requiriendo una habilidad fundamental y de vital importancia (Cole, 2012, p. 553).

Las intervenciones realizadas con urgencia en una UCIN no eximen de la obligación de informar a los padres, sino que aplaza o modifica la forma de hacerlo. La información deberá ser proporcionada tan pronto como haya sido superada la situación de urgencia y, si así lo requieren, en presencia de sus familiares o personas vinculadas a ellos de hecho. Eliminando, de esta manera, cualquier tinte paternalista de la relación sanitaria.

De los textos legales se desprende la existencia de diferentes tipos de información, realizando una especial consideración de la información dirigida a la obtención del consentimiento informado por escrito. Este tipo de consentimiento para técnicas programadas o que acepten demora en una UCIN, requiere que los profesionales sanitarios cuenten con el tiempo suficiente y el espacio adecuado. En el contexto intensivo neonatal se debe evitar la información junto a la incubadora o cuna, sobre todo cuando es relativa a una intervención quirúrgica, a un procedimiento diagnóstico o terapéutico invasivo y en general a cualquier otro procedimiento o técnica que conlleve riesgos o inconvenientes de notoria y previsible repercusión negativa sobre la salud del neonato.

Concretamente, toda la información relacionada con los cuidados realizados al recién nacido deberá ser proporcionada por el enfermero responsable, asumiendo su papel de interlocutor del proceso asistencial en ese momento.

La calidad de la información que reciban los padres del neonato ingresado en una UCIN estará directamente relacionada con la satisfacción percibida ante los cuidados recibidos.

El incumplimiento del derecho a la autonomía de los pacientes supone realizar una práctica profesional de baja calidad técnica, ética y jurídica (Barrio, 2004, p. 102). 


\section{REFERENCIAS BIBLIOGRÁFICAS}

- Barrio CANTAlejo, IM. (2004). "El consentiment informat en infermeria. Ja, peró encara no...", en Bioética \& debate: Tribuna abierta del Institut Borja de Bioética, (38): 101-102.

- Cole, CA. (2012). "Implied consent and nursing practice. Ethical or convenient?", en Nurs Ethics, 19 (4): 554-557. doi: 10.1177/0969733011436028.

- Galán Cortés, J.C. (2014). Responsabilidad Civil Médica (4⿳亠丷⿵冂丶 ed). Navarra: Civitas, Thomson Reuters.

- Osuna CARrillo de Albornoz, E. (2013). "Relación sanitaria e información", en: J.R. SAlCEDo Hernández (Dir.), Derecho y Salud. Estudios de Bioderecho (Comentarios a la Ley 3/2009, de Derechos y Deberes de los Usuarios del Sistema Sanitario de la Región de Murcia) (pp. 83-106). Valencia, Tirant Lo Blanch y Servicio de Publicaciones de la Universidad de Murcia.

- Rodríguez Rabadán, MD. (2017). El Documento de Consentimiento Informado en Enfermería desde el Marco Legal del Principio de Autonomía: La Canalización Venosa Central de Acceso Periférico en Neonatología. (Tesis Doctoral). Facultad de Enfermería, Universidad de Murcia.

- Rubio, R. (2013). "Consentimiento informado", en JR. SALCEDO HERnÁndeZ (Dir.), Derecho y Salud. Estudios de Bioderecho (Comentarios a la Ley 3/2009, de Derechos y Deberes de los Usuarios del Sistema Sanitario de la Región de Murcia) (pp. 129-142), Valencia: Tirant Lo Blanch y Servicio de Publicaciones de la Universidad de Murcia.

- SÁnchez-CARo, J. y Abellán, F. (2003). Derechos y deberes de los pacientes. Ley 41/2002 de 14 de noviembre; consentimiento informado, historia clínica, intimidad e instrucciones previas. Granada: Comares.

- Simón Lorda, P. y JúdeZ GutiérRez, J. (2004). "Consentimiento informado", en D. GRACIA D. Y J. JÚDEZ, (Ed), Ética en la práctica clínica (pp. 33-54). Madrid, España: Triacastela.

\section{NORMATIVA}

- COnstitución EsPañola, Boletín Oficial del Estado no 311, (29-12-1978), pp. 2931329424. Consultada en: https://www.boe.es/diario_boe/txt.php?id=BOE-A-1978-31229

- DECRETO 38/2012, DE 13 DE MARZO, sobre historia clínica y derechos y obligaciones de pacientes y profesionales de la salud en materia de documentación clínica, Boletín Oficial del País Vasco, n65, (29-03-2012). Consultado en: https://www.euskadi.eus/y22bopv/es/bopv2/datos/2012/03/1201512a.shtml

- DECRETO 147/2015, DE 21 DE JULIO, por el que se aprueba la Declaración sobre Derechos y Deberes de las personas en el sistema sanitario de Euskadi, Boletín Oficial del País Vasco $\mathrm{n}^{\circ}$ 146, (04-08-2015). Consultado en: https://www.euskadi.eus/y22bopv/es/bopv2/datos/2015/08/1503424a.shtml

- LEY 14/1986 DE 25 DE ABRIL, General de Sanidad, Boletín Oficial del Estado, nº 102, (2904-1986). Consultada en https://www.boe.es/buscar/doc.php?id=BOE-A-1986-10499

- LEY 1/1992, DE 2 DE JULIO, del Servicio de Salud del Principado de Asturias, Boletín Oficial del Estado, $n^{\circ}$ 211, (02-09-1992), pp. 30265-30273. Consultada en: https://www.boe.es/diario boe/txt.php?id=BOE-A-1992-20655

- LEY 11/1994, DE 26 DE JULIO, de Ordenación Sanitaria de Canarias, Boletín Oficial del Estado, nº 204, (26-08-1994), pp. 27167-27190. Consultada en: 
https://www.boe.es/diario_boe/txt.php?id=BOE-A-1994-19583

- LEY 8/1997, DE 26 DE JUNIO, de Ordenación Sanitaria de Euskadi, Boletín Oficial del País Vasco, $\quad \mathrm{n}^{\mathrm{o}}$ 138, (21-07-1997). Consultada en: https://www.euskadi.eus/y22bopv/es/bopv2/datos/1997/07/9703850a.shtml

- LEY 2/1998 DE 15 DE JUNIO, de Salud de Andalucía, BOE, nº 185, (04-08-1998), pp. 2639226407. Consultada en: https://www.boe.es/diario boe/txt.php?id=BOE-A-1998-18720

- LEY 21/2000 DE 29 DE DICIEMBRE, sobre los derechos de información concernientes a la salud y la autonomía del paciente, y la documentación clínica, Boletín Oficial del Estado n ${ }^{\circ}$ 29, (02-02-2001), pp. 4121-4125. Consultada en:

https://www.boe.es/diario_boe/txt.php?id=BOE-A-2001-2353

- LEY 3/2001, DE 28 DE MAYO, reguladora del consentimiento informado y de la historia clínica de los pacientes, Boletín Oficial del Estado, no 158, pp. 23537-23541. Consultado en: https://www.boe.es/buscar/doc.php?id=BOE-A-2001-12770

- LEY 12/2001, DE 21 DE DICIEMBRE, de Ordenación Sanitaria de la Comunidad de Madrid, BOE, $n^{\circ} 55$ (05-03-2002), pp. 8846-8881. Consultada en: https://www.boe.es/buscar/doc.php?id=BOE-A-2002-4375

- LEY 6/2002, DE 15 DE ABRIL, de Salud de Aragón, BOE, no 121, (21-05-2002), pp. 18061 18079. Consultada en: https://www.boe.es/diario boe/txt.php?id=BOE-A-2002-9667

- LEY 2/2002, DE 17 DE ABRIL, de Salud, BOE, no 106, (03-05-2002), pp. 16210-16237. Consultada en: https://www.boe.es/buscar/doc.php?id=BOE-A-2002-8489

- LEY 41/2002 DE 14 DE NOVIEMBRE, básica reguladora de la autonomía del paciente y de derechos y obligaciones en materia de información y documentación clínica, Boletín Oficial del Estado, $n^{\circ} 274$, (15-11-2002). Recuperada de https://www.boe.es/diario_boe/txt.php?id=BOE-A-2002-22188

- LEY 7/2002, DE 10 DE DICIEMBRE, de Ordenación Sanitaria de Cantabria, Boletín Oficial del Estado, $\quad \mathrm{n}^{\mathrm{o}}$ 6, (7-01-2003), pp. 551 a $577 . \quad$ Consultada en: https://www.boe.es/diario boe/txt.php?id=BOE-A-2003-323

- LEY 5/2003, DE 4 DE ABRIL, de Salud de las Illes Balears, Boletín Oficial del Estado, no 110, (08-05-2003), pp. 17438-17455. Consultada en:

https://www.boe.es/diario_boe/txt.php?id=BOE-A-2003-9336

- LEY 8/2003 DE 8 DE ABRIL, sobre derechos y deberes de las personas en relación con la salud, Boletín Oficial del Estado no 103, (30-04-2003), pp. 16650-16659. Consultada en: https://www.boe.es/diario_boe/txt.php?id=BOE-A-2003-8796

- LEY 3/2005, DE 7 DE MARZO, de modificación de la Ley 3/2001, de 28 de mayo, reguladora del consentimiento informado y de la historia clínica de los pacientes, BOE, $\mathrm{n}^{\circ} 93$ (19-042005), pp. 13364-13368. Consultada en: https://www.boe.es/diario_boe/txt.php?id=BOE-A$\underline{2005-6226}$

- LEY 3/2005, DE 8 DE JULIO, de información sanitaria y autonomía, BOE, no 186, (05-082005), pp. 27513-27524. Consultada en: https://www.boe.es/diario boe/txt.php?id=BOE-A$\underline{2005-13470}$

- LEY 8/2008, DE 10 DE JULIO, de Salud de Galicia, Boletín Oficial del Estado, nº 202, (21-082008), pp. 35080-35115. Consultada en: https://www.boe.es/buscar/doc.php?id=BOE-A2008-14134

- LEY 3/2009 DE 11 DE MAYO, de los Derechos y Deberes de los Usuarios del Sistema Sanitario de la Región de Murcia, BOE, no 34, (09-02-2011), pp. 13608-13639. Consultada en: https://www.boe.es/diario boe/txt.php?id=BOE-A-2011-2493 
- LEY 5/2010, 24 DE JUNIO, sobre derechos y deberes en materia de salud de Castilla-La Mancha, Boletín Oficial del Estado no 248, (13-10-2010), pp. 86329-86352. Consultada en: https://www.boe.es/diario_boe/txt.php?id=BOE-A-2010-15622

- LEY ForAl 17/2010, DE 8 DE NOVIEMBRE, de derechos y deberes de las personas en materia de salud en la Comunidad Foral de Navarra, Boletín Oficial del Estado, no 315 (28-122010), pp. 107659-107693. Consultada en: https://www.boe.es/diario boe/txt.php?id=BOEA-2010-19960

- LEY 10/2011, DE 24 DE MARZO, de derechos y garantías de la dignidad de la persona en el proceso de morir y de la muerte, BOE, $\mathrm{n}^{\circ} 115$, (14-05-2011), pp. 49076 -49093. Consultada en: https://www.boe.es/diario_boe/txt.php?id=BOE-A-2011-8403

- LEY 26/2011 DE 1 DE AGOSTO, de adaptación normativa a la Convención Internacional sobre Derechos de las Personas con Discapacidad, Boletín Oficial del Estado, n 184, (02-082011). Consultada en: https://www.boe.es/buscar/act.php?id=BOE-A-2011-13241

- Ley 10/2014, DE 29 DE DICIEMBRE, de Salud de la Comunitat Valenciana, Boletín Oficial del Estado, $n^{\circ} 35$, (10-02-2015), pp. 11133-11181. Consultada en:

https://www.boe.es/diario boe/txt.php?id=BOE-A-2015-1239

- LEY 1/2015, DE 9 DE FEBRERO, de derechos y garantías de la dignidad de la persona ante el proceso final de su vida, BOE, $\mathrm{n}^{\circ}$ 54, (04-03-2015), pp. 20101-20116. Consultada en: https://www.boe.es/diario_boe/txt.php?id=BOE-A-2015-2295

- LEY 4/2015, DE 23 DE MARZO, de derechos y garantías de la persona en el proceso de morir, BOE, $\mathrm{n}^{\mathrm{o}}$ 96, (22-04-2015), pp. 34962-34981. Consultada en: https://www.boe.es/diario_boe/txt.php?id=BOE-A-2015-4332

- LEY 8/2015 DE 22 DE JULIO, de modificación del sistema de protección a la infancia y a la adolescencia, Boletín Oficial del Estado, $\mathrm{n}^{\circ}$ 175, (23-07-2015), pp. 61871-61889. Consultada en: https://www.boe.es/diario_boe/txt.php?id=BOE-A-2015-8222

- LEY 26/2015 DE 28 DE JULIO, de modificación del sistema de protección a la infancia y a la adolescencia, Boletín Oficial del Estado, $\mathrm{n}^{\circ}$ 180, (29-07-2015), pp. 64544-64613. Consultada en: https://www.boe.es/diario_boe/txt.php?id=BOE-A-2015-8470

- LEY ORGÁNICA 11/2015 DE 21 DE SEPTIEMBRE, para reforzar la protección de las menores y mujeres con capacidad modificada judicialmente en la interrupción voluntaria del embarazo, BOE, n ${ }^{\circ} 227$, (22-10-2015), pp. 83586-83587. Consultada en: https://www.boe.es/diario_boe/txt.php?id=BOE-A-2015-10141

- ORden de 8 dE JUlio de 2009, por la que se dictan instrucciones a los Centros del Sistema Sanitario Público de Andalucía, en relación al procedimiento de Consentimiento Informado, BOJA, no 152, (06-08-2009). Consultada en: https://www.juntadeandalucia.es/boja/2009/152/18 